\title{
Abnormal Contractile Properties of Muscle Fibers Expressing $\beta$-Myosin Heavy Chain Gene Mutations in Patients with Hypertrophic Cardiomyopathy
}

\author{
Edward B. Lankford, ${ }^{\star}$ Neal D. Epstein, ${ }^{\ddagger}$ Lameh Fananapazir, ${ }^{\ddagger}$ and H. Lee Sweeney ${ }^{\S}$ \\ ${ }^{*}$ Cardiovascular Section, Hospital of the University of Pennsylvania, Philadelphia, Pennsylvania 19104-4283; ${ }^{\ddagger}$ Inherited Cardiac \\ Diseases Section, National Heart, Lung and Blood Institute, National Institutes of Health, Bethesda, Maryland 20892; and ${ }^{\S}$ Department \\ of Physiology, University of Pennsylvania, Philadelphia, Pennsylvania 19104-6085
}

\begin{abstract}
Missense mutations in the $\beta$-myosin heavy chain ( $\beta$-MHC) gene cause hypertrophic cardiomyopathy (HCM). As normal and mutant $\beta$-MHCs are expressed in slow-twitch skeletal muscle of $\mathrm{HCM}$ patients, we compared the contractile properties of single slow-twitch muscle fibers from patients with three distinct $\beta$-MHC gene mutations and normal controls. Fibers with the $741^{\text {Gly } \rightarrow \text { Arg }}$ mutation (near the binding site of essential light chain) demonstrated decreased maximum velocity of shortening (39\% of normal) and decreased isometric force generation ( $42 \%$ of normal). Fibers with the $403^{\text {Arg } \rightarrow \text { Gln }}$ mutation (at the actin interface of myosin) showed lowered force/stiffness ratio ( $56 \%$ of normal) and depressed velocity of shortening ( $50 \%$ of normal). Both the $741^{\text {Gly } \rightarrow \text { Arg }}$ and $403^{A r g \rightarrow G i n}$ mutation-containing fibers displayed abnormal force-velocity relationships and reduced power output. Fibers with the $256^{\text {Gly } \rightarrow \text { Glu }}$ mutation (end of ATP-binding pocket) had contractile properties that were indistinguishable from normal. Thus there is variability in the nature and extent of functional impairments in skeletal fibers containing different $\beta$-MHC gene mutations, which may correlate with the severity and penetrance of the disease that results from each mutation. These functional alterations likely constitute the primary stimulus for the cardiac hypertrophy that is characteristic of this disease. (J. Clin. Invest. 1995. 95:1409-1414.) Key words: cardiomyopathy, hypertrophic $\bullet$ myosin, genetics $\bullet$ mutation, genetics $\bullet$ muscle contraction - myocardial contraction
\end{abstract}

\section{Introduction}

Hypertrophic cardiomyopathy (HCM $)^{\prime}$ is a genetic cardiac disease with an autosomal dominant pattern of inheritance, charac-

Address correspondence to H. Lee Sweeney, Department of Physiology, University of Pennsylvania School of Medicine, 37th \& Hamilton Walk, Philadelphia, PA 19104-6085. Phone: 215-898-0485; FAX: 215-5732170.

Received for publication 21 September 1994 and in revised form 14 December 1994.

1. Abbreviations used in this paper: HCM, hypertrophic cardiomyopathy; $\beta$-MHC, $\beta$-myosin heavy chain.

The Journal of Clinical Investigation, Inc.

Volume 95, March 1995, 1409-1414 terized by left ventricular hypertrophy in the absence of another cause for the increased cardiac mass (1). The disease is often associated with disabling symptoms (angina, dyspnea, palpitations, and syncope) and is the most common cause of sudden death in otherwise healthy young individuals (2-4). HCM is genetically heterogeneous (5-13). The phenotype in HCM may be caused by one of several disease genes (5-13). In about a third of HCM kindreds, the disease is caused by 1 of at least 30 distinct mutations in the $\beta$-myosin heavy chain ( $\beta$-MHC) gene located on chromosome $14(5,6,9,10,14-18)$. The mutations consist of single substitutions of highly conserved amino acids and have all been in the myosin head or head-rod junction. It has been shown that the location of the mutation influences the severity and prognosis of the disease in some kindreds $(6,9,10,18)$. For example, the $256^{G l y \rightarrow G l u}$ is associated with a disease penetrance of only $56 \%$ in adults and a benign prognosis (10), but the $403^{\mathrm{Arg} \rightarrow \mathrm{Gln}}$ is associated with a $100 \%$ disease penetrance in both children and adults and is associated with a high incidence of sudden death $(6,9,10)$.

Skeletal muscle contains fast and slow-twitch fibers. The MHC in fast fibers is encoded by genes on chromosome 17. The slow-twitch muscle MHC is identical to ventricular myocardial MHC and is transcribed from the same gene on chromosome 14. Although HCM has been regarded as a primary cardiac disease, it has been demonstrated that the mutant $\beta$-myosin is expressed in slow-twitch skeletal (soleus) muscle of HCM patients with distinct $\beta$-MHC gene mutations $(19,20)$. Furthermore, myosin purified from skeletal muscle obtained from patients with the $403^{\mathrm{Arg} \rightarrow G / n}$ mutation translocates fluorescently labeled actin with reduced velocity in an in vitro motility assay (19). These findings suggest that the study of the contractile properties of slow-twitch skeletal muscle fibers from HCM patients should reveal the primary functional deficits associated with myosin mutations. This in turn, should provide insight into the cascade of adaptations that results ultimately in hypertrophy. The data presented in this study demonstrate that this approach will indeed allow assessment of the impact of myosin mutations on muscle function.

\section{Methods}

Informed consent was obtained in accordance with a study protocol (91-H-0050) approved by the Review Board of the National Heart, Lung and Blood Institute. HCM was defined as left ventricular hypertrophy (maximum left ventricular wall thickness $>15 \mathrm{~mm}$ ) in the absence of another cause of increased mass. In the studied patients, the $\beta$-MHC gene mutations were identified by methods previously described $(9,10)$. 
Soleus muscle biopsies were obtained by open biopsy using sterile technique. Specimens $(\sim 12 \times 3 \times 4 \mathrm{~mm})$ were tied to a wood stick to maintain resting in vivo fiber length, and were immersed and stored at $2^{\circ} \mathrm{C}$ in a solution containing a cocktail of protease inhibitors plus 140 $\mathrm{mM}$ potassium, $4 \mathrm{mM}$ EGTA, $30 \mathrm{mM}$ imidazole, $5 \mathrm{mM}$ ATP, $3 \mathrm{mM}$ magnesium, $100 \mu \mathrm{M}$ leupeptin, and $1 \mathrm{mM} 2$-mercaptoethanol, at $\mathrm{pH}$ 7.1. The bundles remained in this "skinning" solution overnight and, after being changed to a solution containing $50 \%$ glycerol, were shipped at $-20^{\circ} \mathrm{C}$ to Philadelphia. This is a modification of the chemical skinning technique first described by Eastwood et al. (21). On the day of the experiment, fibers were dissected from the muscle bundle and single muscle fibers mounted in " $T$ " clips which had been photoetched from aluminum foil. The fibers were suspended in a relaxing solution ( $\mathrm{pCa}$ 8.0) between a force transducer (Akers AE801; SensoNor, Horten, Norway) and a servo motor system (model 300B; Cambridge Technologies, Inc., Watertown, MA).

The fibers were changed to a fully activating solution, of $\mathrm{pCa} 4.3$, with the following composition (mM): 4 EGTA, 15 creatine phosphate, 1 inorganic phosphate, 3 free magnesium, 100 free potassium, 10 free sodium, $200 \mathrm{U} / \mathrm{ml}$ of creatine kinase, at $\mathrm{pH} 7.1$ and ionic strength of $0.2 \mathrm{M}$. Imidazole was the $\mathrm{pH}$ buffer and was present in concentrations $>30 \mathrm{mM}$. Methyl sulfonate was the major anion and was in excess of $70 \mathrm{mM}$.

Contractile measurements and data collection. The cross-sectional area was estimated by orienting the large diameter of the preparation so that it could be measured under the microscope with an ocular micrometer. The depth of myofibrillar material was determined by focusing through the preparation, noting the depth with the calibrated focus. The area was calculated as the cross-sectional area of an ellipse. Isometric force was measured in the activating ( $\mathrm{pCa} 4.3$ ) solution. The sarcomere uniformity was maintained using the periodic rapid unloaded shortening technique (22). These periodic rapid unloaded shortenings were performed between each perturbation of fiber length in the following protocol.

Control of muscle length was performed using custom software written in our laboratory. The software controlled a programmable filter (9002; Frequency Devices, Haverhill, MA) and digital oscilloscope (model 54600A; Hewlett Packard, Colorado Springs, CO). Data consisting of force and motor position (hence fiber length) were sampled using an A/D board (DT2828; Data Translation, Marlboro, MA) at a frequency of $5 \mathrm{kHz}$. For dynamic stiffness measurements the sampling frequency was $16 \mathrm{kHz}$, and the fiber length change (driving) frequency was $1 \mathrm{kHz}$. Data were saved to disk on an 80486 computer (Microway, Kingston, MA) and analyzed off-line using our custom software.

Maximum velocity of shortening was determined using the Edman slack test (23). The slack test involved first obtaining a steady-state level of isometric tension and then imposing a large and very rapid shortening step. Steps were repeated at varying sizes from $\sim 4$ to $14 \%$ of total fiber length, for a total of nine step sizes. Analysis required determination of the time to the initiation of force redevelopment. Linear regression was performed of the time to force redevelopment (dependent variable) versus step size (independent variable). The maximum velocity $\left(V_{\max }\right)$ of shortening was the reciprocal of the slope of the regression. Fig. 1 demonstrates examples of the raw data traces from six releases from a normal fiber (upper left plot) and five releases from a fiber with the $403^{\mathrm{Arg} \rightarrow G I n}$ mutation (lower left plot). The data and regression are shown in the plots to the right of the corresponding raw data. For the normal fiber (upper right), the regression gave a $V_{\max }$ of 0.93 muscle lengths/s, and for the $403^{\mathrm{Arg} \rightarrow G l n}$-containing fiber (lower right) 0.69 muscle lengths/s. Note that for the purposes of the plot, step size is the ordinate and time the abscissa.

Force-velocity curves were constructed using the load stepping technique. While the permeabilized fiber was maintaining steady-state isometric tension, the load was rapidly (within $0.2 \mathrm{~ms}$ ) changed to a lower constant force using computer feedback control. Within $10 \mathrm{~ms}$, the velocity of shortening was stable. This process was repeated for a total of 16 different loads ranging from 90 to $1 \%$ of isometric force. The force-velocity data were fit using a modification of the equation
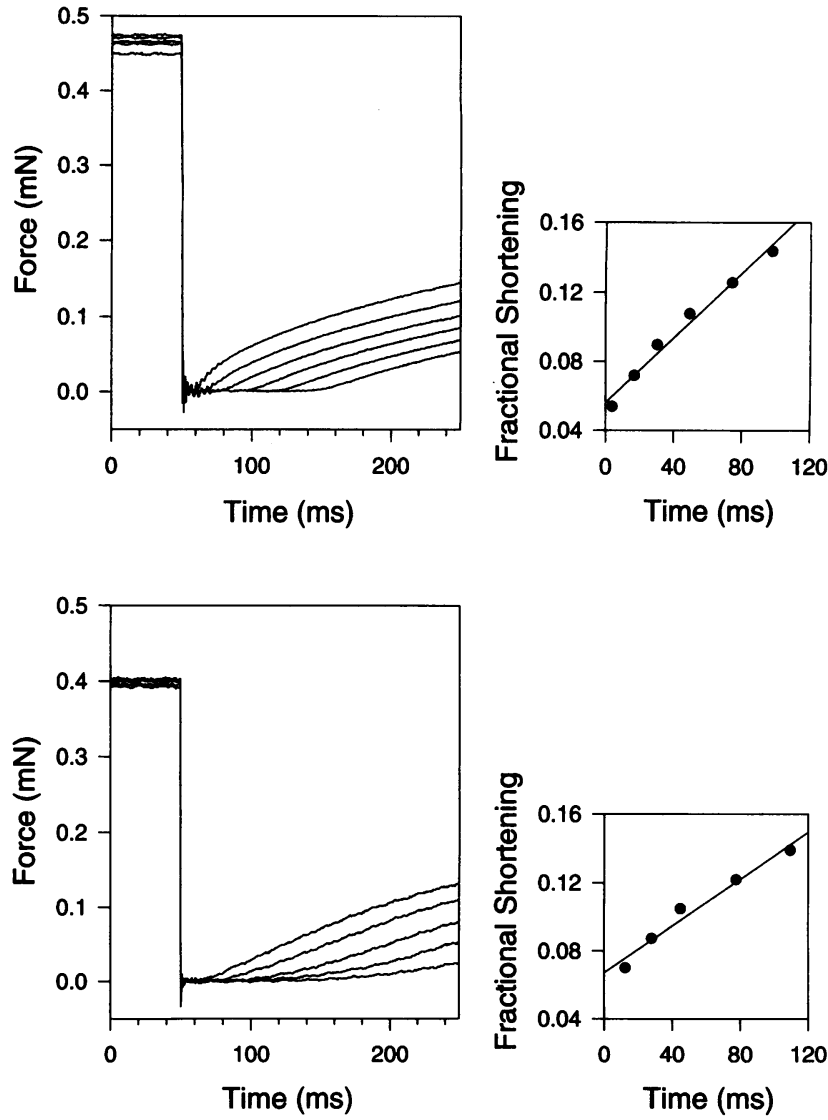

Figure 1. Force traces from a fiber with normal myosin and one containing the $403^{\mathrm{Arg} \rightarrow G l n}$ mutation. Force traces from a normal fiber (upper right plot) from six successively longer releases are shown, and for a fiber with the $403^{\mathrm{Arg} \rightarrow G / n}$ mutation (lower left plot). The time to force redevelopment for each release is determined by custom software and is plotted as the abscissa, and the fractional release step size is the ordinate for each fiber to the right of the corresponding raw data plot. The slope gives $V_{\max }$ and is 0.93 muscle lengths/s for the normal fiber, and 0.69 muscle lengths/s for the fiber containing the $403^{A r g \rightarrow G I n}$ mutation.

originally proposed by A.V. Hill: $\left(\alpha+P / P_{0}\right) V=\alpha V_{\mathrm{m}}\left(1-P / P_{0}\right)$, where $P$ is the load on the fiber, $V$ is the velocity of shortening, $P_{0}$ is the isometric force, $V_{\mathrm{m}}$ is the maximum unloaded velocity of shortening (from the extrapolation of the curve fit to zero load), and $\alpha$ is the curvature of the hyperbolic function, equal to the more commonly presented $\mathrm{a} / \mathrm{P}_{0}$ from the original Hill equation. A custom nonlinear regression routine based on the Levenberg-Marquardt technique was used to curve fit the data (24). Neither maximum isometric force nor maximum unloaded velocity of shortening obtained from the Edman slack test was included in the data set for fitting the force-velocity curve.

Sinusoidal length perturbations were imposed on the muscle of an amplitude of $<0.05 \%$ of total muscle fiber length. Custom software was used for the analysis of the stiffness, and involved taking the fast Fourier transform of the length signal and the force signal. The stiffness was taken as the ratio of the force amplitude to the amplitude of the length change. At the driving frequency of $1 \mathrm{kHz}$ there was generally no viscous component of the impedance ( phase was $\leq 5^{\circ}$ ). The stiffness was normalized to fiber length and cross-sectional area. The isometric force determined from the initial slack tests was divided by the normalized stiffness so calculated. The stiffness gives an indication of the number of tightly bound actin-myosin cross-bridges.

After the contractile experiments, each fiber was removed from the mechanical apparatus and saved for analysis via SDS-glycerol gel elec- 


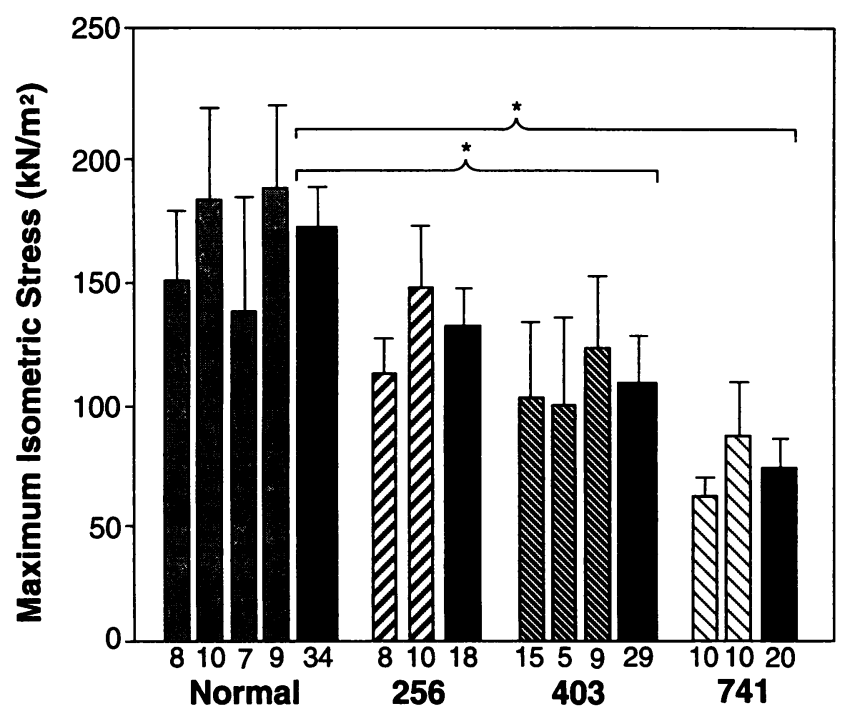

Figure 2. The maximum developed isometric stress. The resulting force with maximum calcium activation is shown and after normalization by cross-sectional area. Each nonsolid bar represents the mean of the fibers studied from each individual, and the wide solid bars show the mean for all fibers studied with the indicated mutation. The numbers below each bar indicate the number of fibers studied from each individual. Error bars are \pm SE.

trophoresis to evaluate $\beta$-MHC composition (25). Only fibers containing $100 \% \beta$-MHC (i.e., no fast MHC) were used in this study. Some fibers have been run on $10 \%$ SDS-glycerol gels to assess the possibility of compensatory changes in contractile protein isoform expression.

Statistical analysis. The results are expressed as mean $\pm 1 \mathrm{SE}$. The maximum unloaded shortening velocities, maximum isometric total and normalized force, and force/stiffness values of fibers from patients with the three distinct mutations are compared with normal controls using the two-tailed Student's $t$ test. A $P$ value of $\leq 0.05$ was considered significant.

\section{Results}

Contractile protein analysis. Gel analysis of each fiber studied indicated that very few fibers did not contain $100 \% \beta$-MHC. Such fibers were eliminated from further analysis. Additional gels were run to analyze possible shifts in isoforms of the myosin light chains, troponin subunits, or tropomyosin in fibers from normals and patients with the $403^{\mathrm{Arg} \rightarrow G l n}$. No shifts were noted in any of the proteins (data not shown).

Cross-sectional area. The cross-sectional area of the remaining (slow-twitch) fibers obtained from patients with the three distinct $\beta$-MHC gene mutations was not significantly different from that of normal control subjects: $256^{\text {Gly } \rightarrow \text { Glu }}$, $2,323 \pm 187 \mu \mathrm{m}^{2} ; 403^{\text {Arg } \rightarrow \text { Gln }}, 2,511 \pm 318 \mu \mathrm{m}^{2} ; 741^{\text {Gl } \rightarrow \text { Arg }}$, $2,339 \pm 217 \mu \mathrm{m}^{2}$; and normal controls, $2,451 \pm 171 \mu \mathrm{m}^{2}$.

Maximum developed isometric force and force/cross-sectional area. The total isometric force generated at maximum activation was analyzed from four normal individuals and seven patients with HCM (data not shown). The four normal individuals had a mean maximum isometric force of $0.34 \pm 0.037 \mathrm{mN}$. The maximum isometric force obtained from the two patients with the $256^{\text {Gly } \rightarrow \text { Glu }}$ mutation was $0.26 \pm 0.033 \mathrm{mN}$ ( $77 \%$ of normal, $P=$ NS). The force from subjects with the $403^{\mathrm{Arg} \rightarrow G l n}$

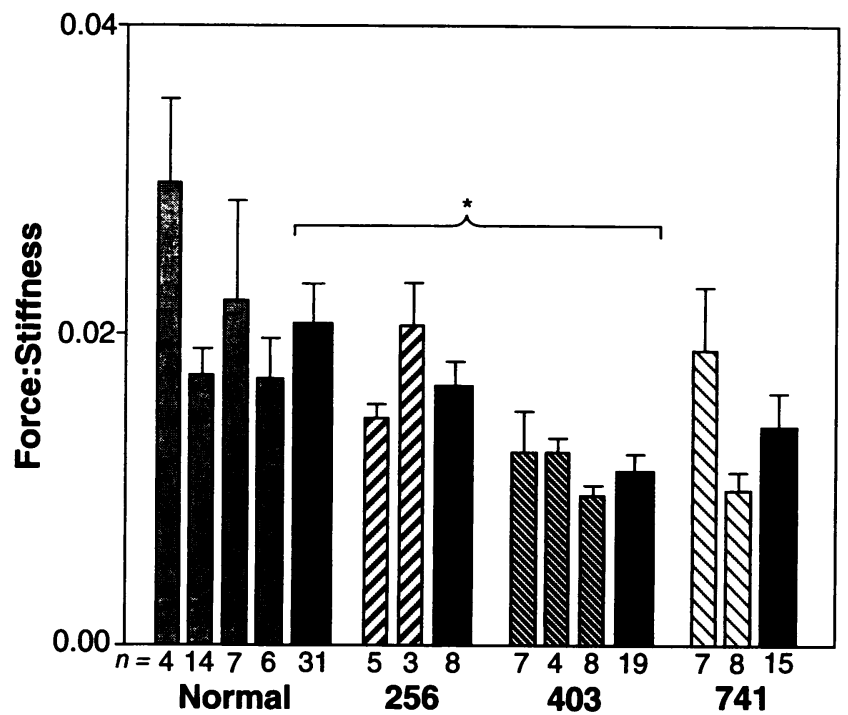

Figure 3. The maximum developed isometric force/stiffness ratio. The ratio of maximum isometric force to stiffness at $1 \mathrm{kHz}$ is shown. Each nonsolid bar indicates the mean of the fibers from each individual studied, and the wide solid black bars are the mean from the fibers with each mutation indicated. The numbers below each bar indicate the number of fibers studied from each individual. Error bars are $\pm \mathrm{SE}$.

mutation was $0.24 \pm 0.046 \mathrm{nN}$ ( $71 \%$ of normal), and it was $0.16 \pm 0.036 \mathrm{nM}(47 \%$ of normal, $P=0.0047)$ for fibers with the $741^{\text {Gl } \rightarrow \text { Arg }}$ mutation.

Fig. 2 shows the maximum isometric force/fiber cross-sectional area for the same fibers as analyzed for total force. The normal maximum isometric stress was $167 \pm 17 \mathrm{kN} / \mathrm{m}^{2}$. The fibers from the patients with the $256^{\text {Gly } \rightarrow \text { Glu }}$ mutation had a maximum normalized force of $128 \pm 16 \mathrm{kN} / \mathrm{m}^{2}(P=\mathrm{NS})$, from the patients with the $403^{A r g \rightarrow G i n}$ mutation $105 \pm 20 \mathrm{kN} / \mathrm{m}^{2}(P$ $=0.016)$, and the $741^{\text {Gly } \rightarrow \text { Arg }} 70.5 \pm 12 \mathrm{kN} / \mathrm{m}^{2}(P=0.005)$.

Force/stiffness ratio. Fiber stiffness is proportional to the number of strongly attached cross-bridges. The force/stiffness ratio can be used as an indication of the force generated per cross-bridge in normal fibers. Fig. 3 shows the results of the force/stiffness ratio. The force/stiffness ratio from normal subjects was $0.020 \pm 0.0021$. Fibers from patients with each mutation and their ratio was $256^{G l y \rightarrow G l u}, 0.017 \pm 0.0016(P=\mathrm{NS})$; $403^{\mathrm{Arg} \rightarrow G l n}, 0.011 \pm 0.0011$ (56\% of normal, $\left.P=0.0015\right)$; $741^{\text {Gly } \rightarrow \text { Arg }}, 0.014 \pm 0.0023(P=\mathrm{NS})$.

Maximum velocity of shortening. The maximum unloaded velocity of shortening, used to quantify the physiologic rate of cross-bridge cycling during fiber activation, is shown in Fig. 4. Normal muscle fibers shortened at a maximum velocity of $1.01 \pm 0.10$ muscle lengths/s. The fibers and their velocities from subjects with the various mutations were as follows: $256^{\text {Gly } \rightarrow \text { Glu }}, 0.90 \pm 0.15 \mathrm{~s}^{-1}(P=\mathrm{NS}) ; 403^{\mathrm{Arg} \rightarrow G / n}, 0.50 \pm 0.072$ $\mathrm{s}^{-1}(P=0.0001) ;$ and $741^{\text {Gl } \rightarrow \text { Arg }}, 0.39 \pm 0.071 \mathrm{~s}^{-1}(P$ $<0.0001)$.

Force-velocity relation. The force-velocity relations provide a more complete characterization of fiber contractile performance and are shown in Fig. $5 A$. The relation from the fibers with the $256^{\text {Gly Glu }}$ mutation was indistinguishable from normal and was omitted for clarity. The composite curves show that the normal fibers were able to shorten with a faster velocity 


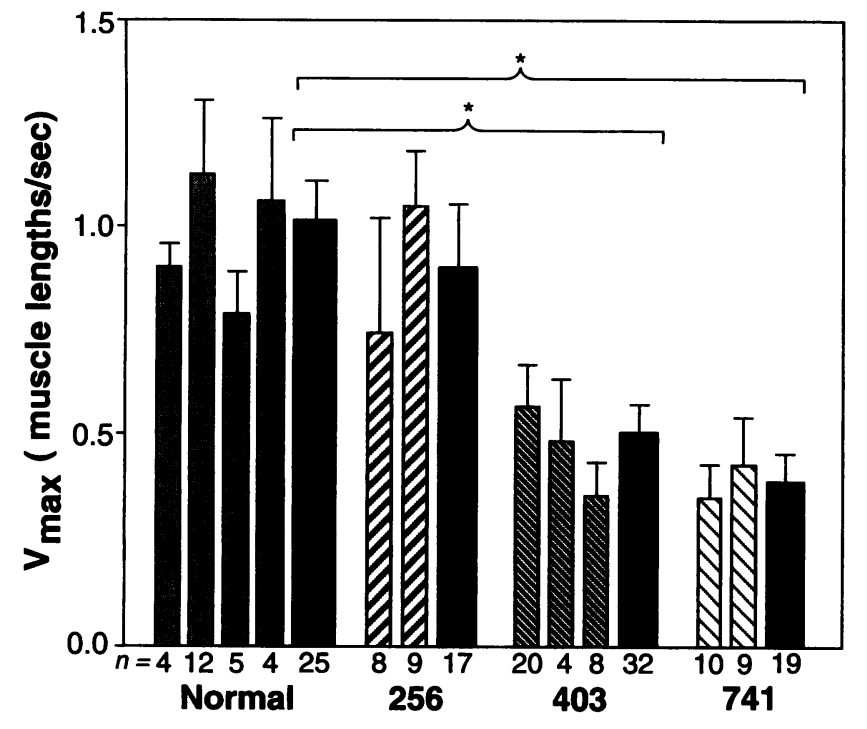

Figure 4. The maximum unloaded velocity of shortening $\left(V_{\max }\right)$. The unloaded velocity of shortening as determined by a series of quick releases of a determined length (Edman slack test) is shown. Each nonsolid bar indicates the mean of the fibers from each individual studied, and the wide solid black bars are the mean from the fibers with each mutation indicated. The numbers below each bar indicate the number of fibers studied from each individual. Error bars are \pm SE.

at each work load compared with fibers from patients with either the $403^{A r g \rightarrow G / n}$ mutation or the $741^{\text {Gly } \rightarrow A r g}$ mutation.

From the curve fit relations, power (force - velocity) was calculated for each fiber, and composite power-load curves were constructed (Fig. $5 \mathrm{~B}$ ). It shows that, as the force-velocity relations would predict, fibers with either the $741^{\text {Gly } \rightarrow \text { Arg }}$ or $403^{A r g \rightarrow G l n}$ mutation had a considerably depressed power output at all loads. Additionally, they must operate over a more restricted load range since they cannot generate as much isometric force.

\section{Discussion}

These experiments demonstrate that mutated MHCs in slowtwitch skeletal muscle have a significant effect on muscle contractile performance. These differences are not attributable to other changes in fibers, such as shifts in contractile protein isoforms or altered levels of protein phosphorylation. Under the conditions of maximal activation at $\mathrm{pCa} 4.3$, alterations in thin filament isoforms have not been shown to affect contractile properties. In any event, no differences in protein isoform expression were detected with SDS-gel electrophoresis. The permeabilization procedure results in complete dephosphorylation of the myosin regulatory light chain (26).

Functional impairment was greatest in the fibers with the $403^{\mathrm{Arg} \rightarrow G / n}$ and $741^{\text {Gly } \rightarrow \text { Arg }}$ mutations, and least in fibers obtained from patients with the $256^{\text {Gly } \rightarrow \text { Glu }}$ mutation. These results are consistent with the clinical parameters of disease penetrance and incidence of sudden death observed in families with the $403^{\mathrm{Arg} \rightarrow G l n}$ and $256^{\text {Gly } \rightarrow \text { Glu }}$ mutations $(8,9)$.

The force-velocity and power output curves shown in Fig. 5 show the distinctive physiologic consequences of the three MHC mutations. The power output curves for the $403^{\mathrm{Arg} \rightarrow G l n}$
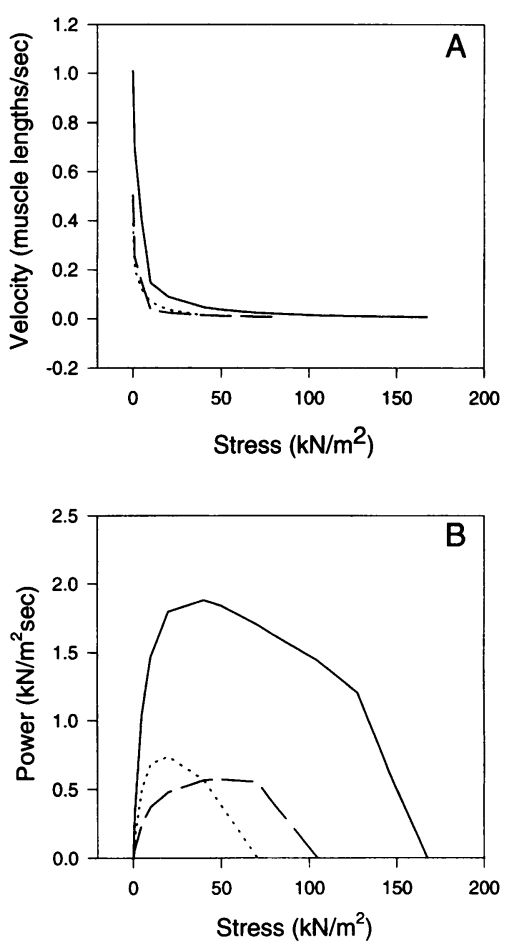

Figure 5. The forcevelocity relation $(A)$ and power output curves $(B)$. The curve fit force-velocity curves were used to construct composite curves from all fibers. Normal fibers are shown with a solid line. Fibers containing the $256^{\text {Gly } \rightarrow \text { Glu }}$ mutation are omitted for clarity. Fibers containing the $403^{\text {Arg } \rightarrow \text { Gln }}$ mutation (long-dashed line) have a lowered velocity of shortening, and the curve is depressed along its entire extent. Fibers containing the $741^{\text {Gly } \rightarrow \text { Arg }}$ mutation (dotted line) have a depressed forcevelocity curve along its entire extent, with depressed intercepts. The calculated power $(B)$ from the force-velocity curves demonstrates the significance of the above force-velocity relation alterations. Lines as above.

and $741^{\text {Gly } \rightarrow \text { Arg }}$ mutations demonstrate severely reduced power over a foreshortened range of loads (force/cross-sectional area) compared with fibers from normal subjects and from patients with the $256^{\text {Gly } \rightarrow \text { Glu }}$ mutation. In addition to reduced force, slowtwitch fibers obtained from patients with these two mutations also exhibited decreased maximum unloaded velocity of shortening.

Although no significant hypertrophy of mutation-containing fibers was seen in this study, we have reported previously that slow-twitch fibers from the soleus muscle from patients with distinct $\beta$-MHC gene mutations are hypertrophied (27). Because in that report muscle biopsy specimens were fixed, sliced in fiber cross-section, and histochemically stained for trichrome and NADH, they provide a more accurate indication of fiber size than do the selective sampling of fibers that we used in this study.

Fibers from patients with the $741^{\text {Gly } \rightarrow \text { Arg }}$ mutation generate less force per cross-sectional area than normal, but fiber stiffness also decreased and force/stiffness remained normal. A decrease in stiffness can result from fewer parallel cross-bridges or a decrease in the stiffness of each cross-bridge (28). From the structure of the myosin head, recently determined via $\mathrm{x}$-ray crystallography (29), the $741^{\text {Gly } \rightarrow \text { Arg }}$ mutation is at the top of the "neck" region of the molecule, near the site of essential light chain binding. One possibility is that a mutation in this region alters the stiffness of the neck and interferes with force transmission from the myosin head to the myosin thick filament, which may normally involve a movement of the neck region (30).

In the case of the $256^{G l y \rightarrow G l u}$ mutation, no significant depression of the force/cross-sectional area was detected, and the 
maximum velocity of shortening was not statistically distinguishable from normal. These patients display the mildest form of the disease of the three patient populations examined, and thus it is reasonable to postulate that the myosin defect is not as functionally severe as for the other two populations examined herein. It is possible that there are more subtle alterations of the mechanical properties than can be detected from these experiments, or that the actual deficit is in some unmeasured property such as fiber contractile efficiency.

The results in Figs. 1-4 demonstrate that fibers containing myosin with the $403^{\mathrm{Arg} \rightarrow G l n}$ mutation have a decreased force/ stiffness ratio and a decreased maximum velocity of shortening. This mutation is at the base of a loop that contributes to the primary actin-binding site and is a part of the major lobe of myosin, whose movement may be necessary for closure of a cleft in the head of the molecule before force production (31). This scheme is speculative, but consistent with an impairment of cleft closure after strong binding of the myosin head to actin, because this would result in a decreased force/stiffness ratio in a muscle fiber and slowed progression through the cross-bridge cycle. This hypothetical effect could result in decreased velocity of shortening. Fig. $5 \mathrm{~B}$ demonstrates a severely decreased power output that has resulted from the abnormal force-velocity relationship depicted in Fig. 5 A.

Quantification of the impact of each myosin mutation on the myosin cross-bridge cycle has not been possible because the fraction of normal to mutant myosin is unknown in the fibers studied. Previous experiments on soleus muscle from the same kindred from which the $403^{\mathrm{Arg} \rightarrow G l n}$ mutated fibers were taken showed that the fraction of the mutated myosin was 40$60 \%$ of total $\beta$-MHC (19). At this time there is no methodology available to differentiate between normal $\beta$-MHC and any mutations found in HCM except the $403^{\mathrm{Arg} \rightarrow \text { Gin }}$. Nevertheless, this limitation does not interfere with the ability to assess the functional impact of the specific mutations on the muscle cells in which the mutant myosins are expressed.

Since the fraction of myosin with the $403^{\mathrm{Arg} \rightarrow \mathrm{Gln}}$ mutation in cardiac muscle of patients was the same as in their slowtwitch skeletal muscle (19), cardiac muscle likely has a similar magnitude of contractile impairment as slow-twitch skeletal fibers. In the heart, the functional adaptations to the impairment are not as successful as in the skeletal muscle, as evidenced by the fact that HCM patients have neither dramatic skeletal muscle hypertrophy nor weakness. This may be because slow-twitch skeletal muscle fibers are used primarily for postural activity. Hypertrophy returns total isometric force toward normal and allows normal function. Additionally, skeletal muscles contain fast fiber types which do not express $\beta$-MHC and can be used to perform the required shortening work of the skeletal muscles, even though the slow-twitch fibers are abnormal. Although the contractile deficits associated with the myosin mutations may provide the stimulus for myocardial hypertrophy, this cannot compensate for slowed shortening velocity and decreased power output as are associated with the $741^{\text {Gly } \rightarrow \text { Arg }}$ and $403^{A r g \rightarrow G l n}$ mutations. Myocardium must perform shortening work to eject blood, so successful cardiac adaptation is more difficult to achieve than for a postural muscle like the soleus. Understanding the adaptation of the heart to myosin functional impairment may lead to successful intervention in this disease.

These results appear in conflict with the historical impression that HCM hearts are hyperdynamic, which has followed from the classic clinical finding in HCM patients of low end systolic volume, and dramatically elevated ventricular ejection fraction. In any heart, the ventricular wall is essentially isovolumic during the cardiac cycle, so with a high myocardial volume and small cavity volume, the geometry dictates a large difference between epicardial and endocardial fractional shortening. HCM hearts appear hypercontractile from clinical assessments because they operate at low volumes and low stress, and not because they demonstrate enhanced myocyte contractile function. This is consistent with recent results of cardiac magnetic resonance imaging in HCM patients, in which regional ventricular myocardial fractional shortening was lower than normal, especially in epicardial segments (32). The various clinical cardiac performance parameters are misleading in HCM primarily because, at the low computed wall stresses that exist in these hearts, function should be considerably greater than it has been found to be.

\section{Acknowledgments}

This work was supported by a grant from the National Institutes of Health (HL-15835). E. B. Lankford was supported by a fellowship from the Southeastern Pennsylvania Affiliate of the American Heart Association. H. L. Sweeney is an Established Investigator of the American Heart Association.

\section{References}

1. Maron, B. J., and S. E. Epstein. 1979. Hypertrophic cardiomyopathy: a discussion of nomenclature. Am. J. Cardiol. 43:1242-1244.

2. Fananapazir, L., D. McAreavey, and N. D. Epstein. 1994. Hypertrophic cardiomyopathy. In Cardiac Electrophysiology, From Cell to Bedside. D. Zipes and J. Jalife, editors. W. B. Saunders Co., Philadelphia. 769-779.

3. Fananapazir, L., A. C. Chang, S. E. Epstein, and D. McAreavey. 1992. Prognostic determinants in hypertrophic cardiomyopathy: prospective evaluation of a therapeutic strategy based on clinical, Holter, hemodynamic and electrophysiologic findings. Circulation. 86:730-740.

4. Dilsizian, V., R. O. Bonow, S. E. Epstein, and L. Fananapazir. 1993. Myocardial ischemia detected by thallium scintigraphy is frequently related to cardiac arrest in young patients with hypertrophic cardiomyopathy. J. Am. Coll. Cardiol. 22:796-804.

5. Geisterfer-Lowrance, A. A. T., S. Kass, G. Tanigawa, H. P. Vosberg, W. McKenna, J. G. Seidman, and C. E. Seidman. 1990. A molecular basis for familial hypertrophic cardiomyopathy: a $\beta$ cardiac myosin heavy chain missense mutation. Cell. 62:999-1006.

6. Watkins, H., A. Rosenzweig, D. S. Hwang, T. Levi, W. McKenna, C. E. Seidman, and J. G. Seidman. 1992. Characteristics and prognostic implications of myosin missense mutations in familial hypertrophic cardiomyopathy. $N$. Engl. J. Med. 326:1108-1114.

7. Schwartz, K., J. Beckmann, C. Dufour, L. Faure, F. Fougerousse, L. Carrier, C. Hengstenberg, D. Cohen, H. P. Vosberg, A. Sacrez, et al. 1992. Exclusion of cardiac myosin heavy chain and actin gene involvement in hypertrophic cardiomyopathy of several French families. Circ. Res. 71:3-8.

8. Epstein, N. D., L. Fananapazir, H. J. Lin, J. Mulvihill, R. White, J.-M. Lalouel, R. P. Lifton, A. W. Nienhuis, and M. Leppert. 1992. Evidence of genetic heterogeneity in five families with familial hypertrophic cardiomyopathy. Circulation. 85:635-647.

9. Epstein, N. D., G. M. Cohn, F. Cyran, and L. Fananapazir. 1992. Differences in clinical expression of hypertrophic cardiomyopathy associated with two distinct mutations in the beta myosin heavy chain gene: a $908^{\text {Leu } \rightarrow \text { Val }}$ mutation and a $403^{\mathrm{Arg} \rightarrow \mathrm{Gin}}$ mutation. Circulation. 86:345-352.

10. Fananapazir, L., and N. D. Epstein. 1994. Genotype-phenotype correlations in hypertrophic cardiomyopathy: insights provided by comparisons of kindreds with distinct and identical $\beta$-myosin heavy chain gene mutations. Circulation. 89:22-32.

11. Thierfelder, L., C. MacRae, H. Watkins, J. Tomfohrde, M. Williams, W. McKenna, K. Bohm, G. Noeske, M. Schlepper, A. Bowcock, et al. 1993. A familial hypertrophic cardiomyopathy locus maps to chromosome 15q2. Proc. Natl. Acad. Sci. USA. 90:6270-6274.

12. Carrier, L., C. Hengstenberg, J. S. Beckmann, P. Guicheney, C. Dufour, J. Bercovici, E. Dausse, I. Berebi-Bertranf, C. Wisnewsky, D. Pulvenis, et al. 1993. Mapping of a novel gene for familial hypertrophic cardiomyopathy to chromosome 11. Nature Genetics. 4:311-313. 
13. Thierfelder, L., H. Watkins, C. MacRae, R. Lamas, W. McKenna, H.-P. Vosberg, J. G. Seidman, and C. E. Seidman. 1994. $\alpha$-Tropomyosin and cardiac troponin $\mathrm{T}$ mutations cause familial hypertrophic cardiomyopathy: a disease of the sarcomere. Cell. 77:701-712.

14. Nishi, H., A. Kimura, H. Harada, H. Toshima, and T. Sasazuki. 1992. Novel missense mutation in cardiac beta myosin heavy chain gene found in a Japanese patient with hypertrophic cardiomyopathy. Biochem. Biophys. Res. Commun. 188:379-387.

15. Coviello, D., P. Spirito, N. Bobola, R. Bertorelli, C. Vecchio, C. E. Seidman, and R. Ravazzolo. 1993. Analysis of the prevalence of 7 cardiac myosin heavy chain gene mutations in 13 Italian families with hypertrophic cardiomyopathy. J. Am. Coll. Cardiol. 21:100a. (Abstr.)

16. Harada, H., A. Kimura, H. Nishi, Y. Koga, T. Sasazuki, and H. Toshima. 1992. Genetic analysis of hypertrophic cardiomyopathy. Circulation. 86:I-591a. (Abstr.)

17. Mares, A., A. J. Marian, Q. T. Yu, G. Z. Czermuszewicz, G. Gooch, T. Tapscott, D. Kelly, J. Towbin, B. Perryman, and R. Roberts. 1992. Genetic screening in 75 families with hypertrophic cardiomyopathy show missense mutations the most common genetic defect. Circulation. 86:I-228a. (Abstr.)

18. Anan, R., G. Gottfried, L. Thierfelder, H. Watkins, W. J. McKenna, S. Solomon, C. Vecchio, H. Shono, S. Nakao, H. Tanaka, et al. 1994. Prognostic implications of novel $\beta$ cardiac myosin heavy chain gene mutations that cause familial hypertrophic cardiomyopathy. J. Clin. Invest. 93:280-285.

19. Cuda, G., L. Fananapazir, W. S. Zhu, J. R. Sellers, and N. D. Epstein. 1993. Skeletal muscle expression and abnormal function of $\beta$-myosin in hypertrophic cardiomyopathy. J. Clin. Invest. 91:2861-2865.

20. Yu, Q. T., J. Ifegwu, A. J. Marlan, A. Mares, R. Hill, M. B. Perryman, L. L. Bachinski, and R. Roberts. 1993. Hypertrophic cardiomyopathy mutation is expressed in messenger RNA of skeletal as well as cardiac muscle. Circulation. 87:406-412.

21. Eastwood, A. B., D. S. Wood, K. L. Bock, and M. M. Sorenson. 1979. Chemically skinned mammalian skeletal muscle. I. The structure of skinned rabbit psoas. Tissue \& Cell. 11:553-566.
22. Brenner, B. 1983. Technique for stabilizing the striation pattern in maximally calcium-activated skinned rabbit psoas fibers. Biophys. J. 41:99-102.

23. Edman, K. A. P. 1979. The velocity of unloaded shortening and its relation to sarcomere length and isometric force in vertebrate muscle fibres. J. Physiol. (Lond.). 291:143-159.

24. Press, W. H., S. A. Teukolsky, W. T. Vetterling, and B. P. Flannery. 1992. Numerical Recipes in C. Second ed. Cambridge University Press. Cambridge. 994 pp.

25. LaFramboise, W. A., M. J. Daood, R. D. Guthrie, P. Moretti, S. Schiaffino, and M. Ontell. 1990. Electrophoretic separation and immunological identification of type $2 \mathrm{X}$ myosin heavy chain in rat skeletal muscle. Biochim. Biophys. Acta. 1035:109-112.

26. Sweeney, H. L., and M. J. Kushmerick. 1985. Phosphorylation of myosin LC-2 in permeabilized rabbit psoas fibers. Am. J. Physiol. 249:C362-C365.

27. Fananapazir, L., M. C. Dalakas, F. Cyran, G. Cohn, and N. D. Epstein. 1993. Missense mutations in the $\beta$-myosin heavy-chain gene cause central core disease in hypertrophic cardiomyopathy. Proc. Natl. Acad. Sci. USA. 90:39933997.

28. Ford, L. E., A. F. Huxley, and R. M. Simmons. 1981. The relation between stiffness and filament overlap in stimulated frog muscle fibres. J. Physiol. (Lond.). 311:219-249.

29. Rayment, I., W. R. Rypniewski, K. Schmidt-Base, R. Smith, D. R. Tomchick, M. M. Benning, D. A. Winkelmann, G. Wesenberg, and H. M. Holden. 1993. Three-dimensional structure of myosin subfragment-1: a molecular motor. Science (Wash. DC). 261:50-58.

30. Lowey, S., G. S. Waller, and K. M. Trybus. 1993. Skeletal muscle myosin light chains are essential for physiological speeds of shortening. Nature (Lond.). 365:454-456.

31. Rayment, I., H. M. Holden, M. Whittaker, C. B. Yohn, M. Lorenz, K. C. Holmes, and R. A. Milligan. 1993. Structure of the actin-myosin complex and its implications for muscle contraction. Science (Wash. DC). 261:58-65.

32. Kramer, C. M., N. Reichek, V. A. Ferrari, T. Theobald, J. Dawson, and L. Axel. 1994. Regional heterogeneity of function in hypertrophic cardiomyopathy. Circulation. 90:186-194. 